\title{
Self perception regarding dental aesthetics, knowledge and attitude of traumatic dental injury and halitosis among people of Aurabani, Sunsari district of Eastern Nepal
}

Dahal $\mathrm{S}^{1}$, Agrawal SK ${ }^{1}$, Shrestha $\mathrm{A}^{2}$, Bhagat $\mathrm{TK}^{3}$

${ }^{1}$ Junior Resident, Department of Public Health Dentistry, CODS, BPKIHS

${ }^{2}$ Additional Professor, Head of Department of Public Health Dentistry, CODS, BPKIHS

${ }^{3}$ Assistnt Professor, Department of Public Health Dentistry, CODS, BPKIHS.

\begin{tabular}{l}
\hline Correspondence \\
Dr.Sirjana Dahal, \\
Junior Resident, \\
Department Of Public Health Den- \\
tistry, \\
College of Dental Surgery, \\
B. P. Koirala Institute Of Health \\
Sciences, Dharan, Nepal. \\
Email: \\
sirjanadahal11@gmail.com \\
DOI: http://dx.doi.org/10.3126/ \\
jcmsn.v11i1.13314
\end{tabular}

\begin{abstract}
Background and Objectives: Increased concern over dental appearance has been observed during childhood and adolescence to early adulthood. The study was conducted with objectives to assess the selfperception of dental aesthetics, knowledge, attitude and management of dental trauma and halitosis. Materials and Methods: Self-administered questionnaire translated in Nepali language consisting of 32 questions was used. The completed questionnaires were analyzed using descriptive statistics. Results: Out of the total study population, $63 \%$ were satisfied with their overall tooth appearance and $71.7 \%$ with their tooth color. Twenty-nine percent had experienced dental trauma. More than half of the study population thought that immediate treatment was required after dental trauma. Fifty seven percent of the villagers had halitosis. Conclusion: The research clearly shows that the people of Aurabani VDC were satisfied with their tooth color and overall tooth appearance; however the knowledge regarding emergency management of dental trauma, the cause and management of halitosis was insufficient.
\end{abstract}

Key words: Dental Aesthetics, Dental Trauma, Halitosis

Citation: Dahal S, Agrawal SK, Shrestha A, Bhagat TK. Self perception regarding dental aesthetics, knowledge and attitude of traumatic dental injury and halitosis among people of Aurabani, Sunsari district of Eastern Nepal. JCMS Nepal. 2015;11(1):6-8.

\begin{abstract}
INTRODUCTION
According to the concept of Oral Health Related Quality Of Life (OHRQoL), good oral health is no longer seen as a mere absence of oral diseases and dysfunction. The definition of OHRQoL includes the absence of negative impacts of oral conditions on social life, and a positive sense of dento-facial self-confidence. ${ }^{1}$ Increased concern over dental appearance has been observed during childhood and adolescence to early adulthood. ${ }^{2}$ The decision to start orthodontic treatment is primarily influenced by such concerns and psychosocial well-being. Social interactions that have a negative effect on self-image, career advancement, and peer-group acceptance have been associated with an unacceptable dental appearance. ${ }^{3}$
\end{abstract}

Dental trauma involves injuries to the tooth and periodontium. Traumatic dental injuries can have a significant impact on the life, affecting both emotionally and physically. ${ }^{4}$ Dental injuries may cause intrusion, extrusion, avulsion, luxation and subluxation of the tooth. Every year a large number of dental injuries are reported especially among children belonging to 7-15 year age group. Studies show that males are injured twice as often as females. The most commonly traumatized tooth is the maxillary central incisor. ${ }^{5,6}$

Halitosis is a general term used to describe unpleasant or offensive odor emanating from the oral cavity. Halitosis may be an important factor during communication; therefore, it may be the origin of concern not only for a possible health condition, but also for frequent psychological alteration leading to social, and personal isolation. ${ }^{7}$ Halitosis is a very common problem in dental patients; in fact most of adult subjects have socially unacceptable breath when they wake up in the morning. ${ }^{8}$ A study performed in Japan involving 
2672 individuals indicated that about $6-23 \%$ of the subjects had oral malodor as measured by Volatile Sulphur Compounds (VSC); higher than 75 parts per billion (ppb) in the expired air, at some period during the day. If these Japanese results reflect the oral malodor in other populations, then the oral malodor would represent a major oral health concern of the public. ${ }^{9}$ Some authors estimate that about $50 \%$ or more of the middle aged individuals had bad breath, due to physiological causes when they wake up in the morning. ${ }^{10}$ Hence, the aim of the study was to assess the knowledge, attitude and practice of dental aesthetics, dental trauma and halitosis among population of Aurabani VDC, Sunsari District of Eastern Nepal.

\section{MATERIALS AND METHODS}

A cross sectional study was done among the people of four wards of Aurabani VDC, Sunsari using convenience sampling technique. Informed consent was taken from villagers before the survey. One hundred and fifty-two villagers who were able to read and write were chosen using a convenience sampling technique. Villagers who were unwilling to take part in study were excluded. A standardized, semi-structured questionnaire was designed on three topics: Dental aesthetics, Dental trauma and Halitosis. ${ }^{5}$ The questionnaire was then translated to Nepali for self-administration. Reliability and validity test was done. The questionnaire consisted of: Personal data, questions regarding dental aesthetics $(\mathrm{n}=8)$ dental trauma $(\mathrm{n}=11)$ and halitosis $(n=13)$. Questionnaires were collected from the villagers and data obtained from the responses was entered in Microsoft Excel Sheet and converted into Statistical Package for Social Sciences version 11.5 software for descriptive statistical analysis.

\section{RESULT}

The total sample included was 152 out of which 78 were male and 74 were female. Results showed that $63 \%$ were satisfied with their overall tooth appearance. It was observed that $71.7 \%$ were satisfied with their tooth color. On assessment of self-perception, nearly $35 \%$ of the study population noticed spacing in between teeth and $30.92 \%$ complained of mal-aligned teeth. Only few of them $(17.10 \%)$ responded to have protruding anterior teeth. About $82 \%$ believed to have caries free anterior teeth but $9.86 \%$ of them complained of fractured anterior teeth. Nearly $13 \%$ responded to hide their teeth while smiling.

Nearly $30 \%$ had experienced dental trauma. Out of 44 people who had experienced trauma, $47.72 \%$ went to dentist but $11.36 \%$ took self-medication.
Majority of the participants $(90.14 \%)$ thought that treatment was needed for dental trauma. When asked about the management, $58.55 \%$ thought that the most appropriate action after avulsion was to stop the bleeding. Nearly half of them (50.66\%) did not know what should be done. Most of them (74.34\%), would contact dentist to seek treatment. More than half $(56.57 \%)$ felt that immediate professional help was needed for avulsion but $50 \%$ did not know that the avulsed tooth could be replanted. Also, 62.5\% did not know about any transportation media. When asked about the avulsion of primary tooth, $67.11 \%$ said that they would not care if any primary tooth got knocked out.

Out of 152 villagers, $57.24 \%$ of them had experienced oral bad breath. Among them, $41.38 \%$ experienced bad breath after awakening and $22.98 \%$ when they were hungry or thirsty. About $42.5 \%$ felt bad breath once a month and $26.43 \%$ every day. When asked, $41.31 \%$ described their bad breath as stinky while $26.47 \%$ described it as bitter. Most of them (70\%) felt that halitosis had affected their social life. Out of 87 people complaining of halitosis, $25.28 \%$ had tongue coating and $50.57 \%$ had problem of xerostomia, $28.7 \%$ were using antibiotics and $18.39 \%$ took antacids. Among those having halitosis, $59.78 \%$ did nothing and $29.88 \%$ had used mouth wash while $24.13 \%$ avoided talking with other people.

\section{DISCUSSION}

This study was conducted to assess the knowledge regarding dental aesthetics, dental trauma and halitosis among the villagers of Aurabani VDC, Sunsari district of Eastern Nepal. Esthetics has become an important aspect of dentistry. Patients have become more aware of dental aesthetics and are seeking treatment to achieve their idea of dental aesthetics. In general, people of Aurabani were satisfied with overall appearance of their teeth (77.63\%). This finding is consistent with study conducted in Simla ${ }^{10}$, India where $60 \%$ of the patients were satisfied with their dental appearance. Many people in Aurabani were happy with the appearance of their tooth colour (71.7\%) and tooth alignment $(69.08 \%)$ whereas in a study done by Geiger SB et al. ${ }^{11}, 89.3 \%$ were not satisfied with their tooth colour, $37.3 \%$ were dissatisfied with their dental appearance followed by poor tooth alignment (23.7\%). In this study, 12.5\% hid their teeth while smiling which was comparable to a study done by Poonam ${ }^{10}$ where $13.6 \%$ reported in doing so, traumatic dental injuries affect approximately one of five school aged children and 
most injuries occur either at home or at school. Parents, teachers and other non-dental professionals can play an important role in management of dental trauma and improving the prognosis. In this study, $28.94 \%$ subjects had experienced dental trauma and $17.10 \%$ unaware of management of avulsed tooth whereas in a study done in Vikarbad town by Anjum $\mathrm{M}^{12}$ in India, $41.4 \%$ experienced dental trauma and $24 \%$ of them were unaware of the management of avulsed tooth. Most of the villagers (74.34\%) consulted the dentist at first which was much higher in comparison to a survey done in Saudi Arabia by Zakirula $M$ et al., ${ }^{5}$ where it was $47 \%$. Almost $3 \%$ of the subjects considered saliva as the best transportation media which was lesser compared to the study done by Zakirulla M.et $\mathrm{al}^{5}$ (11\%).Most adults suffer from genuine halitosis occasionally, while an estimated $10-30 \%$ of population suffers from this problem regularly. ${ }^{13}$ In this study, $57.24 \%$ had experienced oral bad breath out of which $29.83 \%$ of them used mouth wash for subsiding it whereas in a study done in Portugal by Jonas, ${ }^{13} 84 \%$ responded for bad breadth and $55 \%$ used mouthwash. In this study, $41.38 \%$ perceived bad breath after waking up which is lesser than the study done by Almas $\mathrm{K}$ et al. ${ }^{14}$ in King Saud University where it was nearly $63 \%$. Selfmanagement for bad breath was sought by $40.22 \%$ which is comparable to the study done by Almas Ket al. ${ }^{14}$ in King Saud University (38\%).In the present study, $25.29 \%$ had tongue coating which was higher than that of the study done by Almas Ket al. ${ }^{14}$ in King Saud University $20 \%$.

\section{CONCLUSION}

The study clearly implicates that the villagers of Aurabani VDC were satisfied with their tooth colour and overall tooth appearance but the knowledge regarding emergency management of dental trauma was insufficient. Also, the villagers demonstrated lack of knowledge regarding the cause and management of halitosis. Therefore, the development of public awareness programs about dental trauma and halitosis should carry a clear, objective and motivating message in order to make nonprofessionals not only aware of their role in saving teeth in case of accidents but also to make them feel responsible for their overall oral health.
Turkish university students. Eur J Orthod. 2009 Apr;31 (2):168-73. DOI:10.1093/ejo/cjn083.

3. Sardenberg F, Oliveira AC, Paiva SM, Auad SM, Vale MP. Validity and reliability of the Brazilian version of the psychosocial impact of dental aesthetics questionnaire. Eur $\mathbf{J}$ Orthod. 2011 Jun;33(3):270-5. DOI:10.1093/ejo/cjq066.

4. Al-Obaida M. Knowledge and management of traumatic dental injuries in a group of Saudi primary schools teachers. Dent Traumatol. 2010 Aug;26(4):338-41. DOI:10.1111/ j.1600-9657.2010.00894.x.

5. Zakirula M, Togoo RA, Yaseen SM, Al-Shehri DA, AlGhamdi AS, Meer A, et al. Knowledge and attitude of Saudi Arabian school teachers with regards to emergency management of dental trauma. IJCDS May 2011;2(2):24-9.

6. Batra M, Kandwal A, Gupta M, Tangade P, Dany SS, Rajput P. Prevalence of Dental Traumatic Injuries to Permanent Incisors in Indian Children: A Cross- sectional Survey. J Dental Sciences and Oral Rehabilitation. 2014;5 (1):1-4

7. Barzan A, Abeer S. Oral halitosis and oral hygiene practices among dental students. J Bag Coll Dentistry. 2007;19(1):72 -6 .

8. Azodo CC, Onyeagba MI, Odai CD. Does concern about halitosis influence individual's oral hygiene practices? Niger Med J. 2011 Oct;52(4):254-9. DOI:10.4103/03001652.93799 .

9. Iwakura M, Yasuno Y, Shimura M, Sakamoto S. Clinical Characteristics of Halitosis : Differences in Two Patient Groups with Primary and Secondary Complaints of Halitosis. J Dent. Research. 1994 Sep;73(9):1568-74. PMid:7929993.

10.Poonam. Dental Aesthetics and Patient Satisfaction-A hospital based survey. AOSR. 2011;1(1):1-4.

11.Selly BG, Levin L, Gili R. Patients' satisfaction with dental esthetics. J Am Dent Assoc. 2007 Jun;138(6):805-8. DOI:10.14219/jada.archive.2007.0269.

12.Anjum M, Reddy P, Monica M, Yadav K, Abbas I, Reddy A . Knowledge and attitude of primary school teachers in emergency management of dental trauma: A cross-sectional study. Webmed Central Dentistry. 2014;5(10).

13.Jonas CN, Angel MS, Jose M. Halitosis: are dentist being prepared for this challenge? A questionnaire survey in a dental school. Rev port estomatol med dent cirmaxilofac. 2011;52(3):142-6.

14.BaklandLK, Andreasen JO. Dental traumatology: essential diagnosis and treatment planning. Endod Top. 2004 Mar; 7 (1):14-34. DOI:10.1111/j.1601-1546.2004.00059.x.

\section{REFERENCES}

1. Klages U, Bruckner A, Zentner A. Dental aesthetics, selfawareness, and oral health-related quality of life in young adults. Eur J Orthod. 2004;26(5):507-14. DOI:10.1093/ ejo/26.5.507.

2. Hamamci N, Başaran G, Uysal E. Dental Aesthetic Index scores and perception of personal dental appearance among 